\title{
PREDIÇÃO DO METABOLISMO DO CANDIDATO A FÁRMACO CINAMALDEÍDO: Uma abordagem in silico
}

\author{
METABOLISM PREDICTION OF DRUG CANDIDATE CINNAMALDEHYDE: \\ An in silico approach
}

\author{
Ítallo Patrick Sousa Amorim ${ }^{1}$, Edgar Ramos Pestana ${ }^{2}$, Saulo José Figueiredo Mendes ${ }^{3}$
}

\begin{abstract}
RESUMO: O objetivo do presente trabalho foi prever os prováveis sítios de metabolização e os prováveis metabólitos gerados do candidato a fármaco cinamaldeído (CNA) utilizando métodos in silico. Para isso, foram utilizadas duas plataformas virtuais, SMARTCyp e Metaprint2D-React. O SMARTCyp é uma plataforma online de previsão do metabolismo, ele usa algoritmos para fazer a previsão de metabolismo, porém estes algoritmos usam a energia de ativação do Citocromo P450. O Metaprint2D-React é uma plataforma virtual que usa algoritmos para prever os possíveis sítios de metabolismo de uma molécula. A previsão é feita a partir das reações de fase 1 (oxidação e redução) e 2 (conjugação). Para o Metaprint2D-React o principal sítio de metabolismo para o CNA foi a posição carbono 1 , ainda, obteve-se os metabólitos gerados, a reação de redução gerou o composto álcool cinnamílico, já através da hidroxilação obteve-se o ácido cinâmico. O principal local de metabolização no SMARTCyp para o CNA foi o mesmo para o MetaPrint2D-React (Carbono 1), os locais de média e baixíssima chance de metabolização foram divergentes conforme o tipo de enzima do complexo enzimático do citocromo P450. Os resultados obtidos podem nortear outros estudos com o CNA, possibilitando a identificação de metabólitos e atuando no melhoramento químico a partir de diversas estratégias, como, por exemplo, o docking molecular. Ressalta-se que estudos in silico são de grande importância quando associados a outros tipos de estudos na Pesquisa e Desenvolvimento $\mathrm{P} \& \mathrm{D}$ de fármacos.
\end{abstract}

PALAVRAS-CHAVE: toxicologia computacional; toxicocinética; planejamento de fármacos.

ABSTRACT: The aim of the present study was to predict the likely metabolic sites and the likely metabolites generated by the drug candidate cinnamaldehyde (CNA) using in silico methods. For this, two virtual platforms, SMARTCyp and Metaprint2D-React, were used. SMARTCyp is an online platform for predicting metabolism, it uses algorithms to predict metabolism, but these algorithms use cytochrome P450 activation energy. Metaprint2D-React is a virtual platform that uses algorithms to predict the possible sites of metabolism of a molecule. The prediction is made from the reactions of phase 1 (oxidation and reduction) and 2 (conjugation). For the Metaprint2DReact the main metabolism site for the CNA was the carbon 1 position, the metabolites were also obtained, the reduction reaction generated the cinnamyl alcohol compound, and through the hydroxylation cinnamic acid was obtained. The main metabolic site in SMARTCyp for CNA was the same for MetaPrint2D-React (Carbon 1), medium and very low chance of metabolization were divergent according to the enzyme type of the cytochrome P450 enzyme complex. The results obtained can guide other studies with CNA, enabling the identification of metabolites and acting on chemical improvement from several strategies, such as molecular docking. It should be emphasized that in silico studies are of great importance when associated with other types of Research and Development (R\&D) studies in drug.

KEY WORDS: computational toxicology; toxicokinetics; drug planning.

${ }^{1}$ Acadêmico do curso de Farmácia da Universidade Ceuma. Email: itallo215@gmail.com

2 Acadêmico do curso de Farmácia da Universidade Ceuma. Email: edgarpes@gmail.com

${ }^{3}$ Farmacêutico-Bioquímico. Especialista em Gestão da Assistência Farmacêutica/UFSC. Mestre em Biologia Parasitária/Universidade Ceuma. Doutorando em Biodiversidade e Biotecnologia PPG-BIONORTE/Universidade Ceuma. Professor do curso de Farmácia da Universidade Ceuma. E-mail: saulo.mendes@ceuma.br 


\section{INTRODUÇÃO}

A pesquisa com animais é realizada desde os primórdios da ciência. Muitas drogas foram descobertas e experimentadas em modelos in vivo para garantir sua efetividade e segurança. Acredita-se que esses testes em animais aumentam as chances de identificação de drogas promissoras para justificar a utilização em ensaios clínicos em humanos (KNOP \& MARIA, 2016). Contudo, devido ao longo tempo demandado, considerável utilização animal e altos custos, levaram à busca de métodos alternativos, principalmente para a avaliação da toxicidade de candidatos a fármacos utilizando modelos in silico (SANTOS, 2011).

Hoje, o processo de desenvolvimento de medicamentos se esforça para não apenas otimizar a atividade de um candidato a medicamento, mas também estabelecer uma relação entre a farmacodinâmica e a farmacocinética do fármaco (RYDBERG et al., 2010).

Entende-se por modelos in silico (expressão usada com o significado de "executado em computador") métodos computacionais que visam a otimização de moléculas com maior potencial de desenvolvimento. Estes modelos devem ser integrados com os estudos in vitro e in vivo com o intuito de maximizar as chances de sucesso do candidato a fármaco (VAVOUGIOS et al., 2017).

A toxicologia computacional, um tipo de estratégia in silico, vem ganhando grande destaque na Pesquisa e Desenvolvimento $(\mathrm{P} \& \mathrm{D})$ de fármacos, realizados com o auxílio de métodos computacionais e matemáticos para a predição de efeitos adversos e para o melhor entendimento do(s) mecanismo(s) através do(s) qual(is) uma determinada substância provoca o dano (PAPA, 2017). A aplicação da toxicologia computacional encaixa-se à necessidade da indústria farmacêutica de possuir como vantagem a redução de custos e tempo em relação aos modelos experimentais, além da capacidade de processamento de grande quantidade de dados (TOROPOVA, 2017).

Neste contexto, as propriedades farmacocinéticas desempenham um papel muito importante, cerca de $80 \%$ das moléculas presentes no início das pesquisas não alcançam os estudos clínicos, onde $50 \%$ das mesmas tem o insucesso explicado pelas propriedades farmacocinéticas e toxicológicas (PEREIRA, 2007; MODA, 2011). Estudos com as propriedades farmacocinéticas ainda nos estágios inicias das pesquisas e que utilizam a toxicologia computacional leva à eliminação de candidatos não promissores ou pode-se realizar modificações moleculares para melhorar estas propriedades, sendo este processo facilitado e otimizado quando se usa modelos in silico (RAYBURN et al., 2017). 
Uma classe de inegável importância são os anti-inflamatórios, estes compõem o grupo dos medicamentos mais amplamente utilizados por adultos e crianças, com ou sem prescrição médica (PROLUNGATTI et al., 2014). Atualmente, vários candidatos a fármacos anti-inflamatórios têm sido estudados, pela comprovação da sua eficácia e toxicidade. O Cinamaldeído (CNA) é o composto majoritário da casca da canela (45\% 65\%) (CHENG, 1983). O receptor TRPA1 foi identificado na última década como o alvo farmacológico do CNA no organismo, sendo o CNA hoje considerado como um agonista exógeno para este receptor (BANDELL et al., 2004). Nas últimas décadas, as evidências de estudos in vitro e in vivo atribuíram ações imunomoduladoras para o CNA. Recentemente, Mendes, 2016, mostrou que o CNA protege contra a síndrome da resposta inflamatória sistêmica induzida por LPS por mecanismos dependentes e independentes da ativação do TRPA1 (MENDES et al., 2016).

Neste contexto, o presente trabalho tem por objetivo prever os prováveis sítios de metabolização e os prováveis metabólitos gerados do candidato a fármaco CNA utilizando métodos in silico.

\section{METODOLOGIA}

\section{Predição do metabolismo utilizando o software SMARTCyp}

O SMARTCyp é uma plataforma online de previsão do metabolismo, ele usa algoritmos para fazer a previsão de metabolismo, porém estes algoritmos usam a energia de ativação do Citocromo P450 necessária para reagir com uma molécula, que é calculada pela teoria funcional da densidade (DFT, do inglês Density Functional Theory). O candidato a fármaco foi submetido ao software SMARTCyp, disponível em http://smartcyp.sund.ku.dk/. Para interpretação dos resultados, quanto mais baixas as energias de ativação, mais provável que um sítio seja metabolizado (RYDBERG et al., 2010).

\section{Predição do metabolismo utilizando o software MetaPrint2D-React}

O Metaprint2D-React é uma plataforma virtual que usa algoritmos para prever os possíveis sítios de metabolismo de uma molécula. A previsão é feita a partir das reações de fase 1 (oxidação e redução) e 2 (conjugação). Isto é possível através de buscas em data sets que contêm transformações metabólicas já reportadas na literatura científica e que acontecem em posições semelhantes às contidas nas moléculas estudadas com posterior análise estatística dos dados 
encontrados (TARCSAY et al., 2010; ADAMS, 2010; CARLSSON et al, 2010; BRAGA et al, 2012; PIECHOTA et al, 2013; SOUSA et al, 2013). O candidato a fármaco também foi submetido ao software Metaprint2D-React, disponível em http://www-metaprint2d.ch.cam.ac.uk/metaprint2dreact.

\section{RESULTADOS}

No software Metaprint2D-React, obteve-se, primeiramente, os sítios com maior chance de metabolização, estes aparecem em vermelho, os de média chance aparecem em amarelo e os com baixíssima chance aparecem em cinza ou não apresentam coloração. $O$ principal sítio de metabolismo para o CNA foi a posição carbono 1 (Figura 1). Também foi possível verificar a formação de metabólitos gerados a partir do principal local de metabolização e suas respectivas reações químicas. A reação de redução gerou o composto álcool cinnamílico, já através da hidroxilação obteve-se o ácido cinâmico (Figura 2).

Figura 1 - Sítios de metabolismo do Cinamaldeído preditos no MetaPrint2D-React.

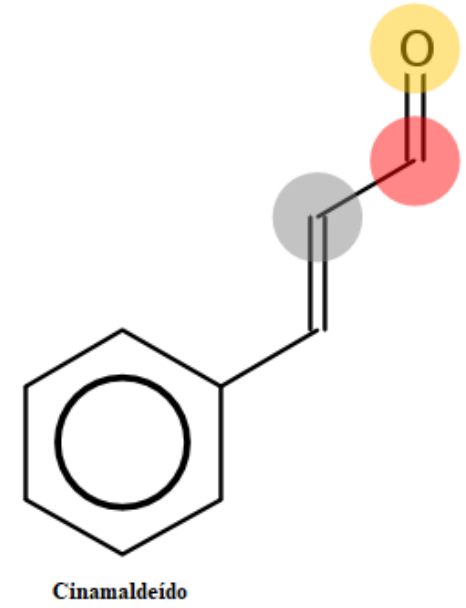


Figura 2- Sítio de metabolismo mais provável do protótipo Cinamaldeído predito no MetaPrint2DReact com os metabólitos gerados e suas respectivas reações químicas.
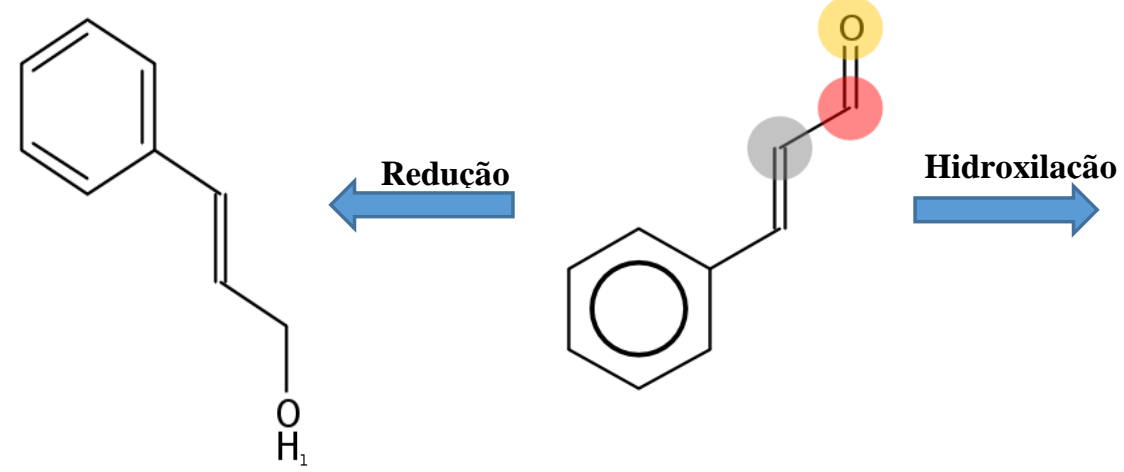

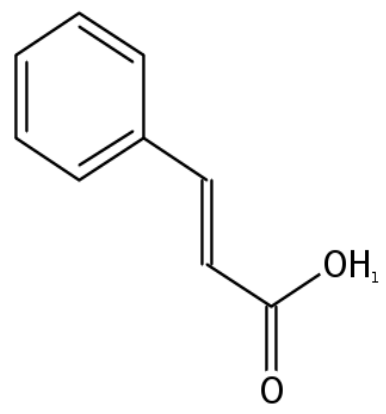

Ácido Cinâmico

O software SMARTCyp apresenta apenas os sítios de metabolização por duas enzimas do citocromo P450, a CYP2C e a CYP2D6, além do método Standard, e assim como no software MetaPrint2D-React classifica a relevância da metabolização por cores. O principal local de metabolização no SMARTCyp para o CNA foi o mesmo para o MetaPrint2D-React (Carbono 1), os locais de média e baixíssima chance de metabolização foram divergentes conforme o tipo de enzima do complexo enzimático do citocromo P450 (Figura 3). 
Figura 3 - Sítios de metabolismo do Cinamaldeído preditos no software SMARTCyp para as enzimas CYP2C e CYP2D6 e também para o modo Standard.

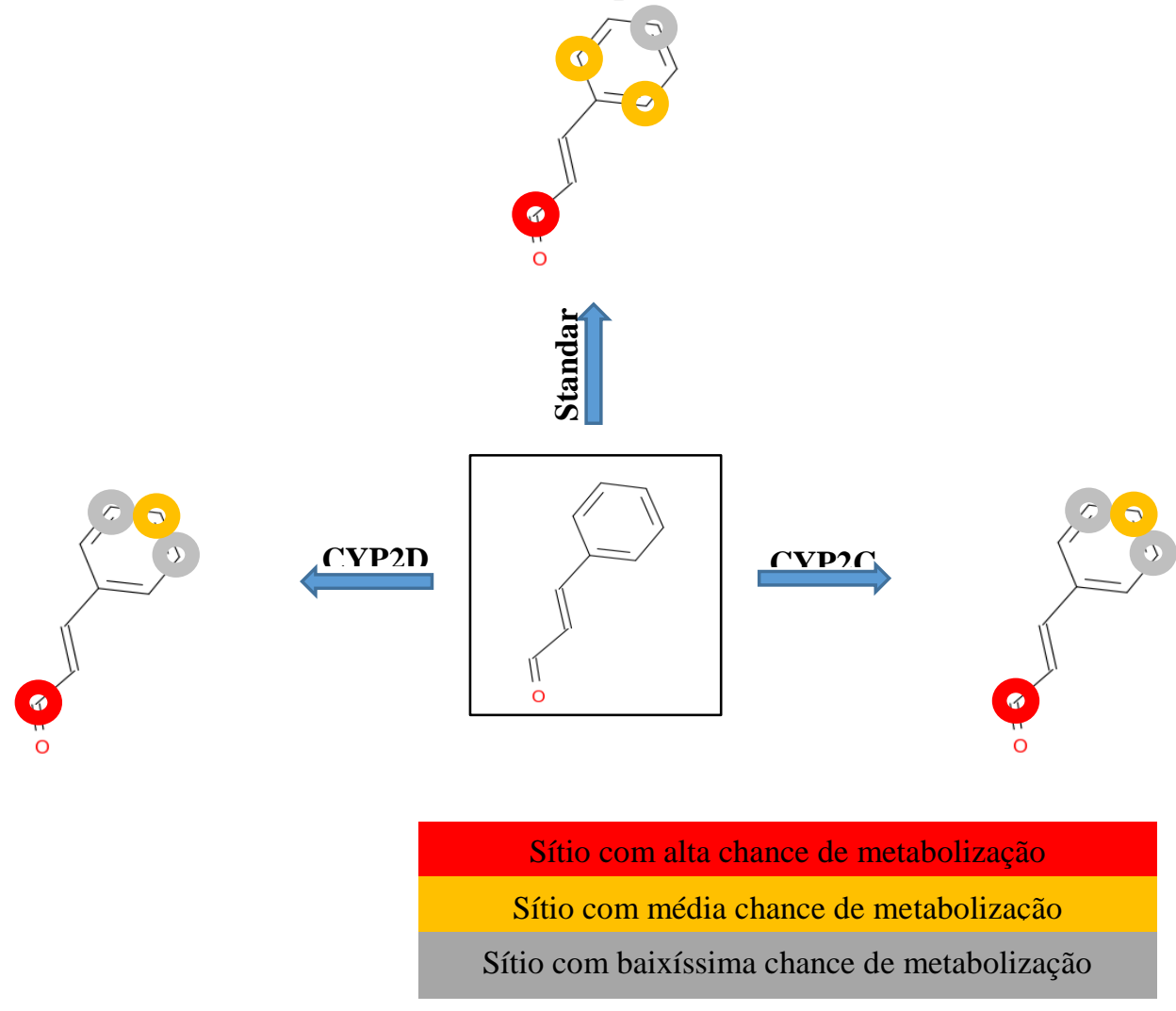

\section{DISCUSSÃO}

Pequenas propriedades farmacocinéticas, bem como os problemas de toxicidade são considerados os principais motivos para encerrar o processo de desenvolvimento de candidatos a medicamentos (FOWLER et al., 2017). Assim, há uma necessidade crescente de métodos robustos de rastreio para fornecer informações precoces sobre absorção, distribuição, metabolismo, excreção e toxicidade (ADMET) de compostos (VALÉRIO, 2012). Dentre as propriedades farmacocinéticas, o metabolismo se destaca por alterar não só a biodisponibilidade como também pode gerar toxicidade (11\% das causas de falha de novos medicamentos) ou que são ativos em receptores não desejados (KIRCHMAIR et al, 2013). A avaliação precoce das propriedades de ADMET podem minimizar o tempo e o custo de triagem e teste, identificando os candidatos mais fortes para o desenvolvimento e rejeitando aqueles com pouca probabilidade de sucesso. O uso aplicado de métodos in silico em novas entidades químicas tonaram-se de grande interesse para as autoridades (JONES et al., 2015). 
Neste trabalho, utilizou-se uma combinação dos softwares SMARTCyp e MetaPrint2DReact, que são duas metodologias gratuitas e de livre acesso, para a predição de sítios de metabolismo e metabólitos do CNA. Isto se justifica devido o SMARTCyp, em contraste com MetaPrint2D-React, não depender de dados já existentes na literatura e, por isso, tem uma menor tendenciosidade nos resultados. Outra vantagem é que esse software prevê o local de metabolismo diretamente da estrutura 2D de uma molécula, sem exigir cálculo de propriedades eletrônicas ou geração de estruturas 3D (RYDBERG et al., 2010). Entretanto, o SMARTCyp não é capaz de prever os metabólitos, o que pode ser de grande importância no $\mathrm{P} \& \mathrm{D}$ de fármacos e, para preencher esta lacuna, foi utilizado o MetaPrint2D-React. Deve-se ressaltar que em testes usando o MetaPrint2D-React é possível prever corretamente os sítios de metabolismo em 70-80\% dos casos (ADAMS, 2010; PIECHOTA et al, 2013).

O principal sítio de metabolização do CNA usando o MetaPrint2D-React foi a posição carbono 6, também foi possível detectar os principais metabólitos gerados através de reações de redução e hidroxilação, um dos compostos gerados foi o ácido cinâmico. Estudos do perfil toxicocinético em ratos mostram que o CNA se revelou instável no sangue e que após administração iv, uma grande fração foi imediatamente oxidado para ácido cinâmico (YUAN et al., 1992). Outros estudos também relatam o potencial do CNA de se metalizar em álcool cinamílico, cinamato de metila e ácido cinâmico no organismo (ZHU et al., 2017). Esses estudos corroboram com os nossos achados, aqui mostramos que os mesmos compostos foram obtidos utilizando apenas estratégias in silico. Apesar dos metabólitos gerados o CNA ainda é considerado um composto natural seguro (LUO et al., 2013; DUGOUA et al., 2007). A Food and Drugs Administration (FDA) recomenda uma dose diária de $1,25 \mathrm{mg} / \mathrm{kg}$.

O conhecimento sobre o tipo de enzimas que serão responsáveis pela metabolização de determinado candidato a fármaco também é de suma importância. O SMARTCyp apresenta os sítios do metabolismo através de enzimas do citocromo P450, a CYP2C e a CYP2D6. A indução enzimática por um fármaco pode alterar concentrações plasmáticas de outro coadministrado e gerar concentrações que não sejam efetivas para este. Se o fármaco coadministrado com indutor enzimático gerar metabólitos a concentração desses produtos irão aumentar e causar efeitos indesejados e até fatais. Por outro lado, a inibição enzimática pode causar acúmulo do eventual fármaco coadministrado, podendo chegar a concentrações tóxicas. Competição de fármacos pela mesma enzima metabolizadora pode diminuir o metabolismo de algum medicamento administrado em conjunto, o que diminui sua excreção e pode atingir níveis tóxicos (WANG et al., 2017). Portanto, métodos computacionais para prever o metabolismo são muito úteis, principalmente para 
candidatos a fármacos com ações em doenças crônicas, pacientes com estas disfunções fazem uso de politerapia e, dessa forma, conhecendo-se as principais vias metabólicas pode-se aperfeiçoar o fármaco em estudo para evitar estes efeitos ou guiar nas orientações ao paciente (SILVA et al., 2014).

Embora abordagens in silico de predição tenha uma boa precisão, um melhor resultado é conseguido quando aliadas a abordagens in vitro e in vivo para que se consiga contornar a maior parte dos problemas, visto que nenhuma das abordagens consegue abranger todas as possibilidades.

\section{CONCLUSÃO}

O presente trabalho utilizou uma metodologia moderna e gratuita para predizer uma importante etapa da farmacocinética de fármacos, o metabolismo, e que pode ser um grande aliado das pesquisas in vitro e in vivo. Os resultados obtidos podem nortear outros estudos com o CNA, possibilitando a identificação de metabólitos e atuando no melhoramento químico a partir de diversas estratégias, como, por exemplo, o docking molecular. Ressalta-se que estudos in silico são de grande importância quando associados a outros tipos de estudos no P\&D de fármacos.

\section{REFERÊNCIAS}

ADAMS, Samuel E. Molecular similarity and xenobiotic metabolism. 2010. Tese de Doutorado. University of Cambridge.

BANDELL, Michael et al. Noxious cold ion channel TRPA1 is activated by pungent compounds and bradykinin. Neuron, v. 41, n. 6, p. 849-857, 2004.

BRAGA, Rodolpho C. et al. Combination of docking, molecular dynamics and quantum mechanical calculations for metabolism prediction of 3, 4-methylenedioxybenzoyl-2-thienylhydrazone. Journal of molecular modeling, v. 18, n. 5, p. 2065-2078, 2012.

CARLSSON, Lars et al. Use of historic metabolic biotransformation data as a means of anticipating metabolic sites using MetaPrint2D and Bioclipse. BMC bioinformatics, v. 11, n. 1, p. 362, 2010.

CHENG, B. C. A review of cinnamon. Zhong Cao Yao, v. 14, n. 134, p. 4762-4765, 1983.

DUGOUA, Jean-Jacques et al. From type 2 diabetes to antioxidant activity: a systematic review of the safety and efficacy of common and cassia cinnamon bark This article is one of a selection of papers published in this special issue (part 1 of 2) on the Safety and Efficacy of Natural Health Products. Canadian journal of physiology and pharmacology, v. 85, n. 9, p. 837-847, 2007. 
FOWLER, Stephen et al. Progress in Prediction and Interpretation of Clinically Relevant Metabolic Drug-Drug Interactions: a Minireview Illustrating Recent Developments and Current Opportunities. Current Pharmacology Reports, v. 3, n. 1, p. 36-49, 2017.

JONES, H. M. et al. Physiologically based pharmacokinetic modeling in drug discovery and development: a pharmaceutical industry perspective. Clinical Pharmacology \& Therapeutics, v. 97, n. 3, p. 247-262, 2015.

KIRCHMAIR, Johannes et al. How do metabolites differ from their parent molecules and how are they excreted? Journal of chemical information and modeling, v. 53, n. 2, p. 354-367, 2013.

KNOP, Luciana Bastianelli; MARIA, Durvanei Augusto. Métodos substitutivos e a experimentação animal: um enfoque inovador. Revista da Sociedade Brasileira de Ciência em Animais de Laboratório, v. 4, n. 2, p. 101-114, 2017.

LUO, Qi et al. Identification of compounds from the water soluble extract of Cinnamomum cassia barks and their inhibitory effects against high-glucose-induced mesangial cells. Molecules, v. 18, n. 9, p. 10930-10943, 2013.

MENDES, Saulo JF et al. Cinnamaldehyde modulates LPS-induced systemic inflammatory response syndrome through TRPA1-dependent and independent mechanisms. International immunopharmacology, v. 34, p. 60-70, 2016.

MODA, Tiago Luiz. Modelagem In silico de propriedades farmacocinéticas para a avaliação de candidatos a novos fármacos. 2011. Tese de Doutorado. Universidade de São Paulo.

PAPA, Ester et al. In Silico Approaches for the Prediction of In Vivo Biotransformation Rates. In: Advances in QSAR Modeling. Springer International Publishing, 2017. p. 425-451.

PEREIRA, Dárcio Gomes. Importância do metabolismo no planejamento de fármacos. Química nova, v. 30, n. 1, p. 171, 2007.

PIECHOTA, Przemyslaw et al. Pragmatic approaches to using computational methods to predict xenobiotic metabolism. Journal of chemical information and modeling, v. 53, n. 6, p. 1282-1293, 2013.

PROLUNGATTI, Camila Nogueira et al. Use of analgesic and anti-inflammatory drugs before assistance in a children's first aid unit. Revista Dor, v. 15, n. 2, p. 96-99, 2014.

RAYBURN, Elizabeth R. et al. FDA-approved drugs that are spermatotoxic in animals and the utility of animal testing for human risk prediction. Journal of Assisted Reproduction and Genetics, p. 1-22, 2017.

RYDBERG, Patrik et al. SMARTCyp: A 2D method for prediction of cytochrome P450-mediated drug metabolism. ACS medicinal chemistry letters, v. 1, n. 3, p. 96-100, 2010.

SANTOS, Carlos Eduardo M. Toxicologia in silico: uma nova abordagem para análise do risco químico. Revista Intertox de Toxicologia, Risco Ambiental e Sociedade, v. 4, n. 1, 2015.

SILVA, Bruno César Mendonça et al. Predição do metabolismo do candidato a protótipo de fármaco anti-inflamatório lqfm002. Revista Eletrônica Faculdade Montes Belos, v. 7, n. 2, 2014.

SOUSA, Mariana C. et al. In silico metabolism studies of dietary flavonoids by CYP1A2 and CYP2C9. Food research international, v. 50, n. 1, p. 102-110, 2013. 
TARCSAY, Ákos; KISS, Róbert; KESERÜ, György M. Site of metabolism prediction on cytochrome P450 2C9: a knowledge-based docking approach. Journal of computer-aided molecular design, v. 24, n. 5, p. 399-408, 2010.

TOROPOVA, M. A. Drug Metabolism as an object of computational analysis by the Monte Carlo method. Current drug metabolism, 2017.

VALERIO JR, Luis G. Application of advanced in silico methods for predictive modeling and information integration. 2012.

VAVOUGIOS, George D. et al. Novel candidate genes of the PARK7 interactome as mediators of apoptosis and acetylation in multiple sclerosis: an in silico analysis. Multiple Sclerosis and

Related Disorders, 2017.

VENKATESH, Srini; LIPPER, Robert A. Role of the development scientist in compound lead selection and optimization. Journal of pharmaceutical sciences, v. 89, n. 2, p. 145-154, 2000.

WANG, Shi-Zhen et al. Strategies and perspectives of assembling multi-enzyme systems. Critical Reviews in Biotechnology, p. 1-14, 2017.

YUAN, J. H. et al. Toxicokinetics of cinnamaldehyde in F344 rats. Food and chemical toxicology, v. 30, n. 12, p. 997-1004, 1992.

ZHU, Ruyuan et al. Cinnamaldehyde in diabetes: A review of pharmacology, pharmacokinetics and safety. Pharmacological Research, 2017. 